\title{
Gastric Balloon Implantation as Part of Morbid Adiposity Therapy Changes the Structure of the Stomach Wall
}

\author{
Maciej Patrzyk ${ }^{a} \quad$ Jenny Sonke ${ }^{b}$ Anne Glitsch ${ }^{a}$ Rebecca Kessler \\ Antje Steveling $^{d} \quad$ Sebastian Lünse $^{a}$ Lars Ivo Partecke $^{\mathrm{e}} \quad$ Claus-Dieter Heidecke $^{a}$ \\ Wolfram Kessler ${ }^{a}$ \\ aDepartment of General, Visceral, Thoracic and Vascular Surgery, University Medical Center Greifswald,

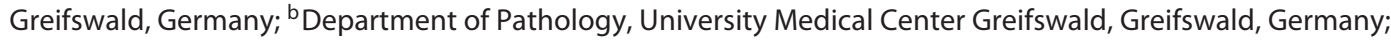 \\ 'Department of Diagnostic Radiology and Neuroradiology, University Medical Center Greifswald, \\ Greifswald, Germany; ${ }^{d}$ Department of Internal Medicine A, University Medical Center Greifswald, \\ Greifswald, Germany; ${ }^{\text {Department }}$ of General, Visceral and Thoracic Surgery, Helios-Klinikum Schleswig, \\ Schleswig, Germany
}

\author{
Keywords \\ Gastric balloon - Sleeve resection - Stomach wall . \\ Hypertrophy of leiomyocytes
}

\begin{abstract}
Purpose: The implantation of a gastric balloon (also known as intragastric balloon) is an established and reversible endoscopic procedure for adiposity therapy. Structural changes of the stomach wall are expected to occur with gastric balloon implantation; however, until now these changes have rarely been investigated. Methods: We compared the histological structure of the stomach wall after gastricsleeve resection in a group of patients following gastric balloon implantation and a group without previous gastric balloon implantation. Results: Following gastric balloon implantation, the tunica muscularis was found to be significantly thicker than without gastric balloon implantation. The enlarging of the tunica muscularis is not caused by hyperplasia of the leiomyocytes, but by hypertrophy of the leiomyocytes and an increase in collagen fibers (fibrosis). Conclusion: A longer-lasting hypertrophy of the tunica muscularis, particularly in the corpus, should be taken into account when surgical treatment follows gastric balloon im-
\end{abstract}

plantation. The staple suture height should be adjusted to the altered tissue composition since reduced tissue elasticity must be expected due to fibrosis.

(C) 2021 The Author(s)

Published by S. Karger AG, Basel

\section{Introduction}

Endoscopic gastric balloon implantation is a temporary measure for reducing weight [1-5], which can be carried out both as part of a weight loss program [6] and in preparation for surgery [1,7-10]. Little is known about the structural changes in the stomach due to gastric balloon implantation. In our view, it would be unusual if a large foreign object (volume 400-700 mL) implanted in a muscular hollow organ for 6 months did not cause changes in the organ's wall structure. However, there is hardly any data available about changes in the stomach wall in the context of gastric balloon treatment. Endoscopic biopsies indicate reversible inflammation of the mucosal lining $[11,12]$. Changes in the main gastric-muscle layer have so far not been investigated or described [13]. Many studies refer to the necessity of using surgical stapling devices of different heights that are basically adjusted to the
(C) 2021 The Author(s)

Published by S. Karger AG, Basel

This is an Open Access article licensed under the Creative Commons Attribution-NonCommercial-4.0 International License (CC BY-NC) (http://www.karger.com/Services/OpenAccessLicense), applicable to the online version of the article only. Usage and distribution for commercial purposes requires written permission.

\section{Wolfram Keßle}

Department of General, Visceral, Thoracic and Vascular Surgery

University Medical Center Greifswald

Ferdinand-Sauerbruch-Str., DE-17475 Greifswald (Germany)

Wolfram.Kessler@med.uni-greifswald.de 
anatomically different stomach wall thickness in the regions of the stomach [14-16]. The overall goal of this study was to examine the structure of the stomach wall of resection specimens from the area of the corpus with and without previous gastric balloon therapy.

\section{Materials and Methods}

Data were collected from the period 2012-2018. We enrolled patients with an initial BMI $>40$ and a medically indicated gastric balloon implantation. There were 12 patients ( 8 females and 4 males) with a successful gastric balloon implantation and subsequent gastric-sleeve creation. One female patient noticed the passing of the gastric balloon via naturalis during the explantation preparation so there was no need for its removal. This day was regarded as explantation day. The specimens of 4 patients with a BMI >40 and gastric-sleeve creation but without previous gastric balloon implantation served as the control group ( 2 females and 2 males) and they underwent a histologic examination.

\section{Gastric Balloon Implantation and Explantation}

The implantation and explantation of the gastric balloon (BIB ${ }^{\mathrm{TM}}$ System Intragastric Balloon, Apollo Endosurgery, Austin, TX, USA) were carried out under sedation with analgesics (Diprivan \pm midazolam) in compliance with the only just-published guidelines in 2015 on sedation in endoscopy [17]. During the calibration (filling) of the gastric balloon, care was taken to ensure a smooth stomach passage with the endoscope and a high volumefilled balloon. The fill volumes of the gastric balloons were approximately $720 \pm 25 \mathrm{~mL}$ (mean \pm SEM). A calibration solely based on the administered volume was not carried out as this seemed to not be explicitly relevant for a successful therapy [18].

\section{Creation of a Gastric Sleeve}

The laparoscopic gastric-sleeve creation took place under general anesthesia. After creating a capnoperitoneum $(14 \mathrm{~mm} \mathrm{Hg}$ ) and exposing the hiatus esophagus, the bursa omentalis was opened approximately $6 \mathrm{~cm}$ oral of the pylorus on the side of the greater curvature; at this point, the omentum majus was removed upwards from the stomach using LigaSure $(10 \mathrm{~mm})$, thus exposing the crus of the diaphragm and the cardial notch. Next, a tension-free gastric sleeve was created by using a $45-\mathrm{mm}$ Endo GIA magazine (tristapler, purple or black magazine) and several 60-mm Endo GIA magazines (tri-stapler, purple magazine) via a 42 Charrière stomach tube (CH42, Dahlhausen, Cologne, Germany). Resected gastric specimens were retrieved, the volume of the resection specimens was ascertained by $\mathrm{NaCl}$ maximum distention, and they were sent to pathology for examination.

\section{Histopathology}

Measurement of Wall Thickness

Each measurement was carried out in the region of the 3 thinnest initial cuts of the mucous membrane by determining the mean value. By choosing the thinnest area of the mucous membrane, the variability induced by an angled cutting of the tissue was reduced. From at least 3 different areas of the proximal, mid$\mathrm{dle}$, and distal gastric-sleeve resection specimens, hematoxylin \& eosin (HE)-stained excised specimens were used for routine diagnostics. The initial cuts were performed along the longitudinal axis of the gastric specimens, i.e., in the corpus and antrum of the resected stomach.
The measurement of the stomach-wall thickness was carried out with camera microscopy (microscope: Leica type 301/371.010; camera: Nikon DS-Fi1) and the NIS-Elements Basic Research Program. The total stomach-wall thickness, stomach-lining thickness, the width of the tunica submucosa, and the tunica muscularis were measured in both groups (i.e., gastric-sleeve creation after gastric balloon implantation and without gastric balloon implantation). Additionally, the ratio of the width of the tunica muscularis to the total thickness of the stomach wall was also determined.

\section{Determination of the Cell Nuclei Density of the Smooth}

Muscles

The cell nuclei density in the area of the diagonally cut bundle of muscles of the tunica muscularis was determined for examining whether the implantation of the gastric balloon had caused hyperplasia of the smooth muscles. The number of nuclei of the smoothmuscle fibers of 5 unit areas (each $0.625 \mathrm{~mm}^{2}$ ) at $\times 400$ magnification was counted. The mean values were calculated from these units.

\section{Fibrosis (Sirius Red Staining Intensity)}

In a third procedure, the connective tissue proportion or fibrosis proliferation of the tunica muscularis and the entire stomach wall were displayed in Sirius Red staining and quantitatively examined. For this purpose, and by using camera microscopy and the NIS Basic Research Program, microscopic photos and the number of red pixel dots (Sirius Red) were captured in the image. The total number of Sirius Red dots was calculated in each identically chosen area. The red parts were thus (in ascending order with the program), multiplied according to their intensity and the number of pixel dots was recorded. The total number of the red portion was the result of the sum of the measured red pixel dots (integral of the detected intensity $\times$ the number of red pixel dots).

\section{Statistics}

Mann-Whitney U tests and Wilcoxon matched-pairs signedranks test were performed using GraphPad Prism v8.0.0 for Windows (GraphPad Software, San Diego, CA, USA; www.graphpad. com).

\section{Results}

At the beginning of the study, the patients had a (mean \pm SEM) BMI of $53.2 \pm 2.3$ in the balloon group and $51.3 \pm 4.6$ in the group without the balloon. There were no differences between these 2 groups in age $(40.3 \pm 3.5$ vs. $50.5 \pm 1.9$ years; $p=0.123)$, gender ( 8 females and 4 males vs. 2 females and 2 males; $p>0.99$ ) and BMI at the time of surgery $(47.3 \pm 1.0$ vs. $51.3 \pm 4.6$; $p=0.123$ ). The balloon had been inside the stomach for approximately 29 weeks (range: $25-39$ weeks; mean \pm SEM: $29.8 \pm 1.2$ weeks). There was an interval of approximately 8 weeks (range: $5-14$ weeks; mean \pm SEM $8.2 \pm 0.8$ weeks) between explantation of the gastric balloon and implementation of the laparoscopic creation of the gastric sleeve. The fill volumes of the gastric balloons were approximately $720 \pm 25 \mathrm{~mL}$.

Regarding the thickness of the stomach wall, the gastric resection specimens examined showed no difference. 
Table 1. Composition of the stomach corpus wall

\begin{tabular}{|c|c|c|c|c|c|c|c|}
\hline & $\begin{array}{l}\text { Total wall } \\
\text { thickness, } \\
\mathrm{mm}\end{array}$ & $\begin{array}{l}\text { Tunica } \\
\text { mucosa, } \\
\mathrm{mm}\end{array}$ & $\begin{array}{l}\text { Tunica } \\
\text { submucosa, } \\
\text { mm }\end{array}$ & $\begin{array}{l}\text { Tunica } \\
\text { muscularis, } \\
\mathrm{mm}\end{array}$ & $\begin{array}{l}\text { Ratio of: } \\
\text { tunica muscularis/ } \\
\text { total wall thickness }\end{array}$ & $\begin{array}{l}\text { Cell nuclei } \\
\text { density }\end{array}$ & $\begin{array}{l}\text { Sirius Red staining } \\
\text { intensity } \\
\text { (tunica muscularis) }\end{array}$ \\
\hline \multicolumn{8}{|l|}{ With balloon } \\
\hline Mean \pm SEM & $4.74 \pm 0.25$ & $1.22 \pm 0.07$ & $0.58 \pm 0.06$ & $2.94 \pm 0.18$ & $0.6212 \pm 0.0183$ & $5.50 \pm 0.24$ & $266,825,000 \pm 11,595,206$ \\
\hline 5 th percentile & 3.62 & & & 2.01 & 0.4850 & 4.6 & $160,000,000$ \\
\hline 25 th percentile & 3.94 & 1.03 & 0.47 & 2.46 & 0.5913 & 5.0 & $257,600,000$ \\
\hline Median & 4.69 & 1.24 & 0.59 & 2.79 & 0.6115 & 5.1 & $267,000,000$ \\
\hline 75th percentile & 5.39 & 1.42 & 0.74 & 3.58 & 0.6723 & 5.8 & $296,825,000$ \\
\hline 95th percentile & 6.43 & & & 3.9 & 0.7210 & 7.4 & $309,500,000$ \\
\hline \multicolumn{8}{|l|}{ Without balloon } \\
\hline Mean \pm SEM & $4.45 \pm 0.52$ & $1.36 \pm 0.36$ & $0.9 \pm 0.26$ & $1.95 \pm 0.33$ & $0.4423 \pm 0.0569$ & $5.85 \pm 0.67$ & $168,725,000 \pm 2,886,860$ \\
\hline 5 th percentile & 2.93 & & & 1.34 & 0.2740 & 4.6 & $162,000,000$ \\
\hline 25th percentile & 3.39 & 0.84 & 0.49 & 1.36 & 0.3263 & 4.7 & $163,525,000$ \\
\hline Median & 4.82 & 1.12 & 0.77 & 1.97 & 0.4845 & 5.9 & $168,400,000$ \\
\hline 75th percentile & 5.14 & 2.12 & 1.44 & 2.52 & 0.5160 & 7.0 & $174,250,000$ \\
\hline 95th percentile & 5.23 & & & 2.53 & 0.5260 & 7.0 & $176,100,000$ \\
\hline Statistics & $\mathrm{ns} ; p>0.99$ & $\mathrm{~ns} ; p=0.86$ & $\mathrm{~ns} ; p=0.32$ & $* p=0.0297$ & $* * p=0.0044$ & ns; $p>0.999$ & ${ }^{*} p=0.01$ \\
\hline
\end{tabular}

ns, not significant; ${ }^{*} p<0.05 ;{ }^{* *} p<0.01$.

Fig. 1. Thickness of the stomach wall (whiskers: 5 th and 95th percentile, median; box: 25th and 75th percentile; $n=12$ [with balloon] vs. $n=4$ [without balloon]). A Stomach wall thickness with/without gastric balloon. The stomach wall thickness has not increased after the gastric balloon implantation $(4.74 \pm 0.25$ vs. $4.45 \pm 0.52 \mathrm{~mm}$; $* p>0.99)$. B Thickness of tunica muscularis with/without gastric balloon. The thickness of the tunica muscularis has significantly increased following the gastric balloon implantation $(2.94 \pm 0.18$ vs. $1.95 \pm$ $0.33 \mathrm{~mm} ; p=0.0297)$. C Ratio of tunica muscularis to stomach wall thickness with/ without gastric balloon. The ratio of the tunica muscularis increases after a gastric balloon $(0.62 \pm 0.02$ vs. $0.44 \pm 0.06$; $p=$ 0.0044). For details, see Table 1.



The mean total wall thickness of the group that had implanted gastric balloons was $4.74 \pm 0.25 \mathrm{~mm}$ and that of the group without previous gastric balloon implantation was $4.45 \pm 0.52 \mathrm{~mm}$. No significant difference between the groups could be verified ( $p>0.99$; Table 1). Figure 1A presents the thickness of the stomach wall with a median of $25 / 75 \%$ percentile (box) and $5 / 95 \%$ percentile (whiskers). However, when examining the stomach wall's individual layers, the main muscle layer of the stomach (tunica muscularis) is clearly thicker $(2.94 \pm 0.18 \mathrm{~mm})$, even a long time after the explantation of the balloon $(8.2 \pm 2.9$ weeks), than in the control group without gastric balloon implantation $(1.95 \pm 0.33 \mathrm{~mm} ; p=0.0297$; Table 1$)$. This is shown in Figure 1B for greater clarity with a percentile and median of $5 / 25 / 75 / 95 \%$. The effect of the muscle layer's increase in size is even more obvious when relating the thickness of the tunica muscularis to the total thickness of the stomach wall (Fig. 1C; Fig. 2; Table 1). Here, in the gastric balloon implantation group, the tunica muscularis takes up approximately $62.12 \pm 1.83 \%$ of the stomach wall compared to $44.23 \pm 5.69 \%(p=0.0044)$ in the group without a previously implanted gastric balloon. Here, too, the effect is illustrated with greater clarity with a percentile and median of 5/25/75/95\% in Figure 1C (and Table 1). The next question was whether the increase in thickness of the tunica muscularis and its proportion of the stomach wall had been caused by cell proliferation or an increase in connective tissue. For this purpose, the 
Fig. 2. A Cross-section of the stomach corpus wall without balloon dilatation, tunica muscularis thickness ( $\}$ ). HE. B Cross-section of the stomach corpus wall following balloon dilatation with clearly increased tunica muscularis propria ( $\}$ ) versus without balloon dilatation. HE. C Cross-section of the stomach corpus wall without balloon dilatation with red-stained connective tissue proportion/fibrosis proliferation (arrows). Sirius Red. D Cross-section of the stomach corpus wall following balloon dilatation with red-stained connective tissue proportion/fibrosis proliferation (arrows) versus without balloon dilatation. Sirius Red. A-D Length of the lower edge of the image: $8 \mathrm{~mm}$.
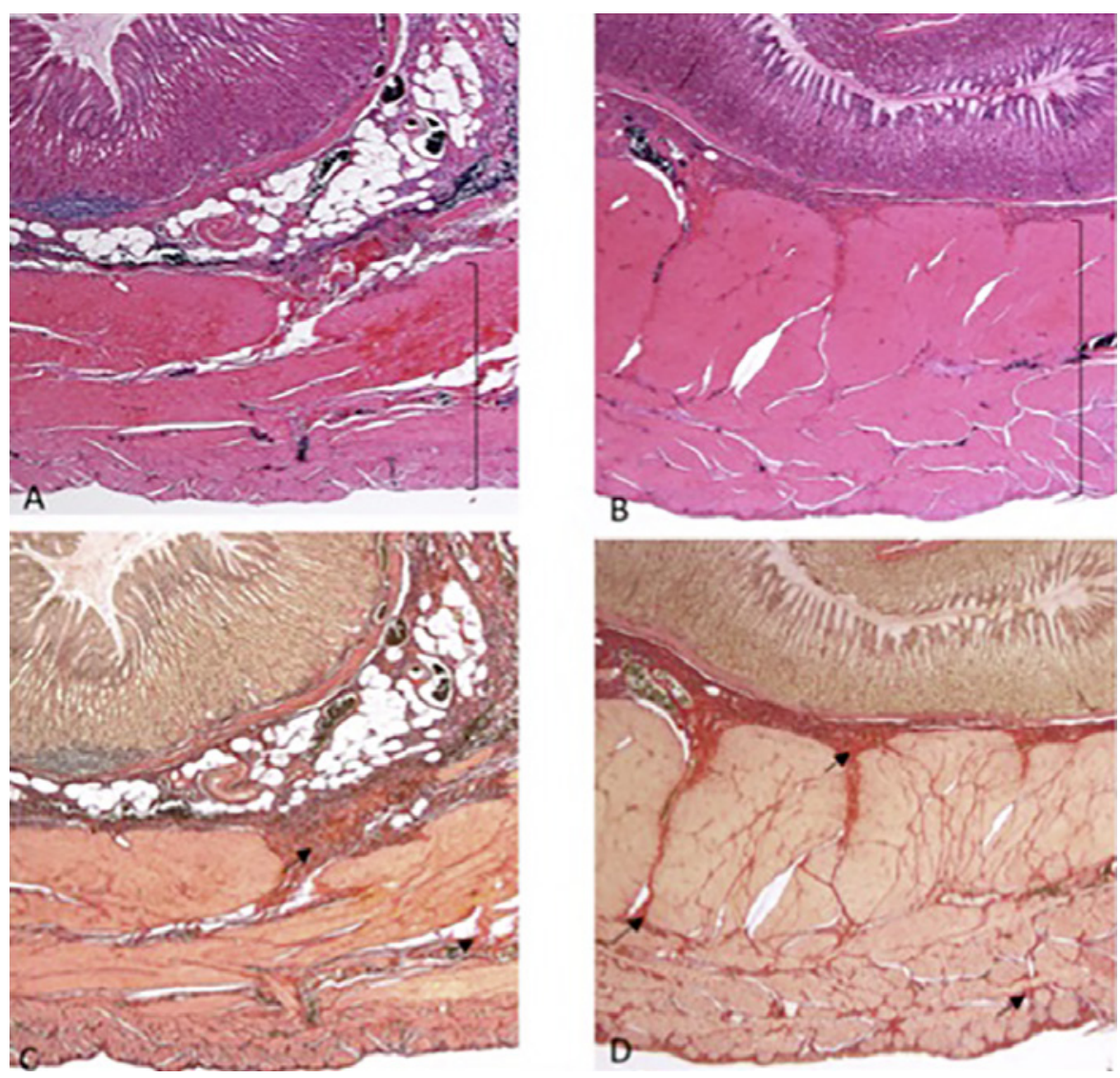

Fig. 3. Specific structure of the stomach wall (whiskers: 5th/95th percentile, median; box: 25 th/75th percentile; ${ }^{*} p<0.05$; $n=12$ [with balloon] vs. $n=4$ [without balloon]). A Display of cell nuclei density with/without gastric balloon. The cell nuclei density with and without gastric balloon cannot be differentiated ( $5.5 \pm 0.24$ vs. $5.58 \pm 0.67 ; p>0.99)$. B Tunica muscularis with/without gastric balloon. Following gastric balloon implantation, patients showed increased intensity of the Sirius Red staining $(266,825,000 \pm 11,595,206$ vs. $168,725,000 \pm 2,886,860, p=0.0132)$. For details, see Table 1.

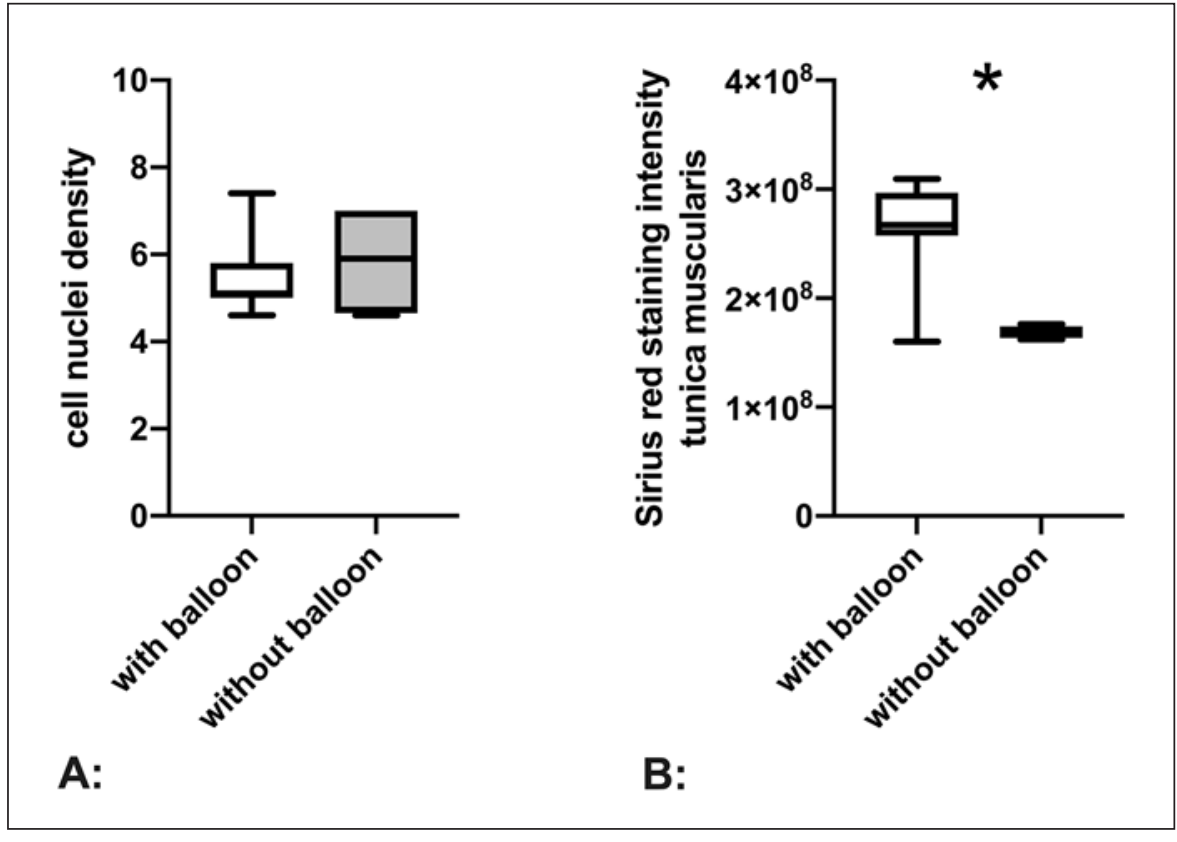

cell nuclei density in the stomach wall was determined. The density of the stomach wall's cell nuclei did not increase in the group of former gastric balloon patients (5.5 \pm 0.24 vs. $5.58 \pm 0.67$ without balloon; $p>0.9999$ ), illustrated in Figure $3 \mathrm{~A}$ with percentile details (see above). It must therefore be concluded that the increase in tunica muscularis thickness was not caused by proliferation or infiltration of cells. Sirius Red staining of the connective tissue section exhibited a significantly higher proportion of red per tunica muscularis surface in patients after gas- 
tric balloon implantation than the staining intensity of the group without gastric balloon implantation (Fig. 2; Fig. 3B; Table 1; $p=0.0132$ ), corresponding to an increased proportion of collagen making up the tissue volume. These results support the hypothesis that this is most likely due to tissue hypertrophy and fibrosis rather than hyperplasia.

\section{Discussion}

The implantation of a gastric balloon is an established and effective intervention in the therapy of adiposity [15], where, depending on the indication, bariatric surgical interventions [1,7-9] as well as other surgical therapies (e.g., hernia repair [8] and liver transplantation [10]) may follow. However, gastric balloon implantation is not recommended as a noncritical standard procedure for weight reduction prior to bariatric interventions $[19,20]$. The gastric balloon implantation itself, i.e., as a standalone adiposity therapy, is inferior to bariatric surgery in the long run $[21,22]$. It is a procedure where complications have been observed, such as esophageal perforation and stomach muscle rupture including bowel obstruction due to deflation of the balloon $[2,23]$. Pancreatitis $[24,25]$ has also been known to occur after gastric balloon implantation. Following balloon implantation, inflammation of the gastric mucosa [26], sometimes culminating in life-threatening bleeding ulcers, has been reported [27]. Histologically, inflammatory alterations of the gastric mucosa infiltrated with CD3-positive T cells and an increased cell division reaction (Ki-67) could be observed during gastric balloon explantation [11]; these were not present at the time of the balloon implantation, meaning that this therapy might also be of immunological relevance. Reversible and mostly inflammatory alterations of the gastric mucosa were described over a period of 2 weeks following a gastric balloon explantation [12]. This is why we recommended an appropriate period of time between gastric balloon explantation and any subsequent surgical therapy [12]. As our study verified changes in the stomach wall structure (Fig. 1B, C; Fig. 2A-D; Fig. 3B; Table 1) even 8 weeks after the explantation of the gastric balloon, the time period between balloon explantation and surgical therapy should be extended to beyond 2 weeks.

The stomach wall's generally varying thickness profile [14-16] implies the recommendation to use surgical stapling devices with adjusted surgical-staple heights when creating a gastric sleeve [14-16]. The thickest stomach wall is situated in the antrum and the thinnest stomach wall in the fundus [14-16]. In some segments, there is a difference in height of approximately $1 \mathrm{~mm}$ [28] between the fundus and antrum. The distribution of our model's stomach wall thickness did not apply to the anatomical position within the organ but focused on the change triggered by the gastric balloon implantation. In doing so, no changes in the stomach wall thickness could be detected in our patient group after gastric balloon implantation (Fig. 1A). The fact that our wall thickness measurements after fixation (Fig. 1A) of $4.74 \pm 0.25$ versus $4.45 \pm 0.52$ $\mathrm{mm}$ were in the upper range of the wall thickness in fresh specimens (antrum: $3.12 \mathrm{~mm}$ in males vs. $3.09 \mathrm{~mm}$ in females) described by Huang and Gagner [14] might have been caused by the fixation. However, it may also have been linked to the fact that they used compression to determine the stomach wall thickness $[14,28]$.

A research paper by Boeker et al. [13] describes the tissue structure of the stomach wall in the fundus and compares it to the leakage rate as well as other parameters, but without identifying clear differences regarding the detailed stomach wall structure [13]. Yazar et al. [29] determined the stomach wall thickness by histologic examination and ultrasound, and then correlated the results with existing inflammation. In doing so, an increased thickness of the mucosa wall (mucosa + muscularis mucosa + submucosa) might be correlated with inflammation; of note, they did not determine the thickness of the tunica muscularis [29]. Our histologic examinations of the tunica mucosa did not detect a change in the thickness of the stomach wall of the group after gastric balloon therapy (Table 1), which, according to Yazar et al. [29] contradicts the existence of gastritis at the time of our examination. However, it is likely that the gastritis described within the context of a balloon explantation [11] and which was still verifiable 14 days after the balloon explantation [12], had completely healed after an additional 6 weeks (the time point in our study).

It was a different situation with the changes in the tunica muscularis. Here, we were able to determine an increase in thickness after gastric balloon therapy compared to in the patients without gastric balloon implantation (Fig. 1B), also illustrated by the higher proportion of the tunica muscularis in the thickness of the stomach wall (Fig. 1C; Fig. 2A, B). This effect could be verified 8 weeks after removing the irritation from the stomach (gastric balloon). As the increase in tunica muscularis thickness is not explained by an increase in the number of cells (Fig. 3A; Table 1), but by the increase in collagen fibers (Fig. 2C, D; Fig. 3B; Table 1), we have to assume this involved a longer-term hypertrophy of the tissue.

\section{Conclusion}

To the best of our knowledge, our study presents the first histologic examination of the stomach wall structure following a gastric balloon implantation. Whilst stomach 
wall thickness was not influenced by the preceding gastric balloon implantation, the altered tissue composition, with hypertrophy and fibrosis, is suspected to lead to reduced elasticity and compressibility of the tissue. In the case of subsequent laparoscopic gastric-sleeve creation, the altered structure of the stomach, particularly in the corpus, should be taken into account when choosing the heights of the staple sutures.

\section{Statement of Ethics}

All procedures performed were in accordance with the ethics standards of the institutional research committee and the 1964 Helsinki Declaration and its later amendments or comparable standards. The Ethics Commission of the Greifswald University Medical Center approved the study (registry No. BB24/12). Written informed consent was obtained from all individual participants included in the study.

\section{Conflict of Interest Statement}

The authors declare that they have no conflicts of interest.

\section{Funding Sources}

There was no funding.

\section{Author Contributions}

M.P. and C.-D.H. designed the work, interpreted the data, and revised the work critically. J.S. acquired and analyzed the data, drafted the work, and revised the work critically. A.G. designed the study, acquired the data, and revised the work critically. R.K. interpreted the data and revised the work critically. A.S. acquired and analyzed the data and revised the work critically. S.L. acquired and interpreted the data and revised the work critically. I.P. designed the study, interpreted the data, and revised the work critically. W.K. designed the work, acquired, analyzed, and interpreted the data, and wrote the manuscript.

\section{References}

1 Ahmed HO, Ezzat RF. Quality of life of obese patients after treatment with the insertion of intra-gastric balloon versus Atkins diet in Sulaimani Governorate, Kurdistan Region, Iraq. Ann Med Surg (Lond). 2018 Nov;37: $42-6$

2 Stavrou G, Tsaousi G, Kotzampassi K. Lifethreatening visceral complications after intragastric balloon insertion: is the device, the patient or the doctor to blame? Endosc Int Open. 2019 Feb;7(2):E122-9.

3 Martins Fernandes FAJ, Carvalho GL, Lima DL, Rao P, Shadduck PP, Montandon ID, et al. Intragastric Balloon for Overweight $\mathrm{Pa}$ tients. JSLS. 2016;20:e2015.00107.

4 Abu Dayyeh BK, Kumar N, Edmundowicz SA, Jonnalagadda S, Larsen M, Sullivan S, et al.; ASGE Bariatric Endoscopy Task Force and ASGE Technology Committee. ASGE Bariatric Endoscopy Task Force systematic review and meta-analysis assessing the ASGE PIVI thresholds for adopting endoscopic bariatric therapies. Gastrointest Endosc. 2015 Sep;82(3):425-38.e5.

5 Mathus-Vliegen EM, Alders PR, Chuttani R, Scherpenisse J. Outcomes of intragastric balloon placements in a private practice setting. Endoscopy. 2015 Apr;47(4):302-7.

6 Dastis NS, François E, Deviere J, Hittelet A, Ilah Mehdi A, Barea M, et al. Intragastric balloon for weight loss: results in 100 individuals followed for at least 2.5 years. Endoscopy. 2009 Jul;41(7):575-80.

7 Zerrweck C, Maunoury V, Caiazzo R, Branche J, Dezfoulian G, Bulois P, et al. Preoperative weight loss with intragastric balloon decreases the risk of significant adverse outcomes of laparoscopic gastric bypass in super-super obese patients. Obes Surg. 2012; 22:777-82.
8 Dąbrowiecki S, Szczęsny W, Popławski C, Sosnowski D. Intragastric Balloon (BIB system) in the treatment of obesity and preparation of patients for surgery - own experience and literature review. Pol Przegl Chir. 2011 Apr;83(4):181-7.

9 Göttig S, Daskalakis M, Weiner S, Weiner RA. Analysis of safety and efficacy of intragastric balloon in extremely obese patients. Obes Surg. 2009 Jun;19(6):677-83.

10 Choudhary NS, Puri R, Saraf N, Saigal S, Kumar N, Rai R, et al. Intragastric balloon as a novel modality for weight loss in patients with cirrhosis and morbid obesity awaiting liver transplantation. Indian J Gastroenterol. 2016 Mar;35(2):113-6.

11 Atef E, Zalata KR, Atef H, Abdel-Hamid AAM. Increased Proliferative Activity Accompanies the Local Inflammatory Response of Gastric Mucosa After Intragastric Balloon Insertion. Dig Dis Sci. 2016;61:3498-505.

12 Joffe OY, Molnar IM, Tarasyuk TV, Tsyura YP, Stetsenko OP, Kryvopustov MS. [Morphological changes of gastric mucosa after insertion of intragastric balloon]. Klin Khir. 2015 Oct;(10):70-2.

13 Boeker C, Mall J, Reetz C, Yamac K, Wilkens L, Stroh C, et al. Laparoscopic Sleeve Gastrectomy: Investigation of Fundus Wall Thickness and Staple Height-an Observational Cohort Study: Fundus Wall Thickness and Leaks. Obes Surg. 2017 Dec;27(12): 3209-14.

14 Huang R, Gagner M. A Thickness Calibration Device Is Needed to Determine Staple Height and Avoid Leaks in Laparoscopic Sleeve Gastrectomy. Obes Surg. 2015 Dec; 25(12):2360-7.

15 Barski K, Binda A, Kudlicka E, Jaworski P, Tarnowski W. Gastric wall thickness and stapling in laparoscopic sleeve gastrectomy - a literature review. Wideochir Inne Tech Malo Inwazyjne. $2018 \mathrm{Mar} ; 13(1): 122-7$.
16 Susmallian S, Goitein D, Barnea R, Raziel A. Correct Evaluation of Gastric Wall Thickness May Support a Change in Staplers' Size When Performing Sleeve Gastrectomy. Isr Med Assoc J. 2017 Jun;19(6):351-4.

17 Riphaus A, Wehrmann T, Hausmann J, Weber B, von Delius S, Jung M, et al.; Gesellschaft Politics and Law in Health Care (GPRG). [S3-guidelines "sedation in gastrointestinal endoscopy" 2014 (AWMF register no. 021/014)]. Z Gastroenterol. 2015 Aug; 53(8):802-42.

18 Kumar N, Bazerbachi F, Rustagi T, McCarty TR, Thompson CC, Galvao Neto MP, et al. The Influence of the Orbera Intragastric Balloon Filling Volumes on Weight Loss, Tolerability, and Adverse Events: A Systematic Review and Meta-Analysis. Obes Surg. 2017 Sep;27(9):2272-8.

19 Leeman MF, Ward C, Duxbury M, de Beaux AC, Tulloh B. The intra-gastric balloon for pre-operative weight loss in bariatric surgery: is it worthwhile? Obes Surg. 2013 Aug; 23(8):1262-5.

20 Adrianzén Vargas M, Cassinello Fernández N, Ortega Serrano J. Preoperative weight loss in patients with indication of bariatric surgery: which is the best method? Nutr Hosp. 2011 Nov-Dec;26(6):1227-30.

21 Dang JT, Switzer NJ, Sun WY, Raghavji F, Birch DW, Karmali S. Evaluating the safety of intragastric balloon: An analysis of the Metabolic and Bariatric Surgery Accreditation and Quality Improvement Program. Surg Obes Relat Dis. 2018 Sep;14(9):1340-7.

22 Vyas D, Deshpande K, Pandya Y. Advances in endoscopic balloon therapy for weight loss and its limitations. World J Gastroenterol. 2017 Nov;23(44):7813-7. 
23 Twardzik M, Wiewiora M, Glück M, Piecuch J. Mechanical intestinal obstruction caused by displacement of a stomach balloon - case report. Wideochir Inne Tech Malo Inwazyjne. 2018 Jun;13(2):278-81.

24 Gore N, Ravindran P, Chan DL, Das K, Cosman PH. Pancreatitis from intra-gastric balloon insertion: case report and literature review. Int J Surg Case Rep. 2018;45:79-82.

25 Said F, Robert S, Mansour EK. Pancreatitis and intragastric balloon insertion. Surg Obes Relat Dis. 2016;12:e33-4.
26 Albanopoulos K, Natoudi M, Sioka E, Leandros E, Zacharoulis D. Gastritis might be considered as a technical factor affecting laparoscopic sleeve gastrectomy. J Surg Case Rep. 2018;2018:rjy169.

27 Mojkowska A, Gazdzinski S, Fraczek M, Wylezol M. Gastric Ulcer Hemorrhage - a Potential Life-Threatening Complication of Intragastric Balloon Treatment of Obesity. Obes Facts. 2017;10:153-9.
28 van Rutte PW, Naagen BJ, Spek M, Jakimowicz JJ, Nienhuijs SW. Gastric Wall Thickness in Sleeve Gastrectomy Patients: Thickness Variation of the Gastric Wall. Surg Technol Int. 2015 Nov;27:123-8.

29 Yazar FM, Baykara M, Karaağaç M, Bülbüloğlu E. The Role of Conventional Ultrasonography in the Evaluation of Antrum Wall Thickness in Obese Patients. Obes Surg. 2016 Dec;26(12):2995-3000 